\title{
GROWTH OF HAAR SERIES ON THE DYADIC RATIONALS AND UNIQUENESS
}

\author{
WILLIAM R. WADE
}

ABSTRACT. We show that uniqueness holds for Haar series which satisfy a certain pointwise growth condition on a certain countable set. It was previously held that this growth condition had to hold at every point in the interval $[0,1]$.

The purpose of this paper is to correct a long-standing misconception (see [1], [2] and [4]). In particular, we obtain the following result as a corollary to Theorem 2:

Theorem 1. Suppose that $S(x)=\Sigma_{k=0}^{\infty} a_{k} \chi_{k}(x)$ is a Haar series which converges except on a countable set $E \subseteq[0,1]$ to a function $f(x)$ in $L^{1}[0,1]$. Let $D \subseteq[0,1]$ represent the set of dyadic rationals and suppose further that

$$
\lim _{n \rightarrow \infty} \frac{a_{n} \chi_{n}\left(x_{0}\right)}{n}=0 \quad \text { for } x_{0} \in E \cup D .
$$

Then $S$ is the Haar-Fourier series of $f$.

It was previously held that (1) must hold for every $x_{0} \in[0,1][1, \mathrm{p}$. 1405]. We found it curious that all examples to show (1) could not be relaxed at a single point were examples involving dyadic rationals. Once we were convinced that dyadic rationals were in some sense special, we were even more surprised that no new techniques were needed to establish this fact.

We now state our main result and indicate its proof.

Theorem 2. Suppose that $S=\Sigma_{k=0}^{\infty} a_{k} \chi_{k}(x)$ is a Haar series which converges almost everywhere to a Denjoy integrable function $f$ on $[0,1]$. Suppose further that for some countable subset $E \subseteq[0,1]$,

$$
\limsup _{n \rightarrow \infty}\left|S_{n}(x)\right|<\infty \quad \text { for } x \notin E
$$

and that (1) holds for each $x_{0} \in E \cup D$. Then $S$ is the Denjoy-Haar-Fourier series of $f$.

Received by the editors April 25, 1974.

AMS (MOS) subject classifications (1970). Primary 42A56, 42A48.

Key words and phrases. Haar functions, Denjoy integrable. 
As in [5], given any nonnegative integer $k=2^{n}+m\left(0 \leq m<2^{n}\right)$ and $i=1$ or 2 denote the open interval

$$
\left((2 m+(i-1)) / 2^{n+1},(2 m+i) / 2^{n+1}\right)
$$

by $\Delta(i, k)$, and its closure by $\Delta^{*}(i, k)$.

Lemma 1. Let $S$ be a Haar series satisfying (1) for some fixed $x_{0}$ and suppose that there are integers $k_{0} \geq 0$ and $i_{0}=1$ or 2 such that

$$
s_{k_{0}+1}(x) \neq 0 \text { for } x \in \Delta\left(i_{0}, k_{0}\right) \text {. }
$$

Then there are integers $k_{0}^{\prime} \geq 0$ and $i_{0}^{\prime}=1$ or 2 such that

and

$$
S_{k_{0}^{\prime}}(x) \neq 0 \text { for } x \in \Delta\left(i_{0}^{\prime}, k_{0}^{\prime}\right)
$$

$$
x_{0} \notin \Delta^{*}\left(i_{0}^{\prime}, k_{0}^{\prime}\right) \text {. }
$$

Proof. Suppose the lemma is false. Then there is some sequence of intervals $\Delta\left(i_{0}, k_{0}\right), \Delta\left(i_{1}^{\prime}, N_{1}\right), \Delta\left(i_{2}^{\prime}, N_{2}\right), \cdots$ such that $x_{0}$ lies in the intersection of the ir closures and

$$
S_{N_{k}+1}(x) \equiv 0 \quad \text { for } x \in \Delta\left(i_{k}^{\prime}, N_{k}\right), \quad k=1,2, \cdots .
$$

This is precisely the situation at the top of p. 224 in [4]. The remainder of the proof follows precisely as in [4].

Lemma 2。 Let $f$ be Denjoy integrable, $T$ be its Haar-Fourier series and suppose that $S(x)=\Sigma a_{k} \chi_{k}(x)$ is any Haar series which converges to $f$ almost everywhere. Suppose further that there exist integers $k_{0}^{\prime} \geq 0$ and $i_{0}^{\prime}=1$ or 2 such that

$$
S_{k_{0}^{\prime}+1}(x) \neq T_{k_{0}^{\prime}+1}(x) \text { for } x \in \Delta\left(i_{0}^{\prime}, k_{0}^{\prime}\right) .
$$

Then given any $M>0$ there are integers $k_{1} \geq 0$ and $i_{1}=1$ or 2 such that $\Delta\left(i_{1}, k_{1}\right) \subset \Delta\left(i_{0}^{\prime}, k_{0}^{\prime}\right)$,

$$
S_{k_{1}+1}(x) \neq T_{k_{1}+1}(x) \quad \text { for } x \in \Delta\left(i_{1}, k_{1}\right)
$$

and

$$
\left|S_{k_{1}+1}(x)\right|>M \text { for } x \in \Delta\left(i_{1}, k_{1}\right) \text {. }
$$

This result is known [2, p. 333] for Haar series $S$ which converge to $f$. off a countable set. Straightforward modifications of that proof will 
establish this lemma. The crucial point is that no growth condition is involved (compare with [5, Lemma 4] and [4, Lemma 1]).

The proof of Theorem 2 proceeds as follows. Let $E=\left\{z_{1}, z_{2}, \ldots\right\}$ and suppose that $S$ is not the Haar-Fourier series $T$ of $f$. Then there is at least one pair of integers $i_{0}, k_{0}$ such that

$$
S_{k_{0}+1}(x)-T_{k_{0}+1}(x) \neq 0 \text { for } x \in \Delta\left(i_{0}, k_{0}\right) \text {. }
$$

Now $T$ satisfies (1) everywhere (see [2]) so we can apply Lemma 1 to the Haar series $S-T$. Since the endpoints of $\Delta\left(i_{0}, k_{0}\right)$ are dyadic rationals, we apply this lemma three times to find integers $i_{0}^{\prime}, k_{0}^{\prime}$ such that (6) holds and such that $z_{1} \notin \Delta *\left(i_{0}^{\prime}, k_{0}^{\prime}\right) \subseteq \Delta\left(i_{0}, k_{0}\right)$. Now by Lemma 2 there are integers $i_{1}, k_{1}$ such that (7) holds, $\left|S_{k_{1}+1}(x)\right|>1$ for $x \in \Delta\left(i_{1}, k_{1}\right)$ and $\Delta\left(i_{1}, k_{1}\right) \subseteq \Delta\left(i_{0}^{\prime}, k_{0}^{\prime}\right)$. In particular $\Delta *\left(i_{1}, k_{1}\right) \subseteq \Delta^{*}\left(i_{0}^{\prime}, k_{0}^{\prime}\right) \subseteq \Delta\left(i_{0}, k_{0}\right)$.

Continuing in this manner we can choose a sequence of intervals $\Delta\left(i_{1}, k_{1}\right), \Delta\left(i_{2}, k_{2}\right), \ldots$ such that

$$
\begin{gathered}
z_{n} \notin \Delta^{*}\left(i_{n}, k_{n}\right), \quad n=1,2, \cdots, \\
\Delta^{*}\left(i_{n}, k_{n}\right) \subseteq \Delta\left(i_{n-1}, k_{n-1}\right), \quad n=1,2, \cdots,
\end{gathered}
$$

and

$$
\left|S_{k_{n}+1}(x)\right|>n \quad \text { for } x \in \Delta\left(i_{n}, k_{n}\right)
$$

$n=1,2, \ldots$. By $(10)$ we let $\xi \in \bigcap_{n=1}^{\infty} \Delta\left(i_{n}, k_{n}\right)$ and observe that (11) and (2) imply that $\xi \in E$. However, since this is incompatible with (9) our proof is established by contradiction.

We close this paper by drawing attention to a similar situation for Walsh series. Indeed, although the pointwise growth condition used in [3] is believed to be necessary at every point in the group $2^{\omega}$, all the examples involve $2^{\omega}$ analogues of dyadic rationals. One cannot help but wonder if this growth condition is also superfluous on the dyadic irrationals.

\section{BIBLIOGR APHY}

1. F. G. Arutjunjan and A. A. Talaljan, Uniqueness of series in Haar and Walsh systems, Izv. Akad. Nauk SSSR Ser. Mat. 28 (1964), 1391-1408. (Russian) MR $30 \# 2282$.

2. F. G. Arutjunjan, Reconstruction of the coefficients of series with respect to Haar and Walsh systems convergent to functions integrable in the sen se of Denjoy, Izv. Akad. Nauk SSSR Ser. Mat. 30 (1966), 325-344. (Russian) MR 33 \#1650. 
3. R. B. Crittenden and V. L. Shapiro, Sets of uniqueness on the group $2^{\omega}$, Ann. of Math. (2) 81 (1965), 550-564. MR 31 \#3783.

4. W. R. Wade, Uniqueness theory for Cesaro summable Haar series, Duke Math. J. 38 (1971), 221-227.

5. Uniqueness of Haar series which are $(C, 1)$ summable to Denjoy integrable functions, Trans. Amer. Math. Soc. 176 (1973), 489-498. MR 47 \#704.

6. R. J. Lindahl, A differentiation theorem for functions defined on the dyadic rationals, Proc. Amer. Math. Soc. 30 (1971), 349-352. MR $44 \# 1774$.

DEPARTMENT OF MATHEMATICS, UNIVERSITY OF TENNESSEE, KNOXVILLE, TENNESSEE 37916

PROCEEDINGS OF THE

AME RICAN MATHEMATICAL SOCIETY

Volume 50, July 1975

\section{$L_{p, q}$ MODULARS $^{*}$}

\section{HIDEGORO NAKANO}

ABSTRACT. For $1<p \leq q$, a convex modular $m$ on a linear space $S$ is called an $L_{p, q}$ modular if $\operatorname{Min}_{r=p, q} \xi^{r} m(x) \leq m(\xi x) \leq \operatorname{Max}_{r=p, q} \xi^{r} m(x)$ for $\xi>0$ and $x \in S$. We generalize the Minkowski inequality and the Hölder inequality for $L_{p, q}$ modulars.

For $1<p \leq q$, a convex modular $m$ on a linear space $S$ is called an $L_{p, q}$ modular if

and

$$
\xi^{p} m(x) \leq m(\xi x) \leq \xi^{q} m(x) \text { for } \xi \geq 1 \text { and } x \in S,
$$

$$
\xi^{p} m(x) \geq m(\xi x) \geq \xi^{q} m(x) \text { for } 0<\xi \leq 1 \text { and } x \in S .
$$

An $L_{p, q}$ modular is $p$-homogeneous if $p=q$, as defined in [3]. We generalized the Minkowski inequality and the Hölder inequality for $p$-homogeneous modu-

Received by the editors July 11, 1972 and, in revised form, September 27, 1973. AMS (MOS) subject classifications (1970). Primary 46B99.

Key words and phrases. Normed linear spaces, Banach spaces.

* This paper is being published posthumously. Galley proofs were corrected by Professor Kazumi Nakano, Department of Mathematics, SUNY at Brockport, Brockport, New York 14420. 